\title{
Periodic, Irrational and Transcendental Numbers in the Factorial Series
}

\author{
István Lénárt \\ Eötvös Loránd University, Budapest, Hungary \\ Email: lenarti@caesar.elte.hu, h12572len@ella.hu
}

How to cite this paper: Lénárt, I. (2022) Periodic, Irrational and Transcendental Numbers in the Factorial Series. Journal of $A p$ plied Mathematics and Physics, 10, 558-575. https://doi.org/10.4236/jamp.2022.102041

Received: January 22, 2022

Accepted: February 22, 2022

Published: February 25, 2022

Copyright $\odot 2022$ by author(s) and Scientific Research Publishing Inc. This work is licensed under the Creative Commons Attribution International License (CC BY 4.0).

http://creativecommons.org/licenses/by/4.0/

\begin{abstract}
The main aim of the article is to investigate the irrational and transcendental properties of certain real numbers by means of the factorial series and the factorial number system. The difference between the factorial series and the factorial system is that the factorial series does not set an upper bound at a given place after the radix point, while in the factorial system $(i-1)$ is the maximal possible value for $r_{i}$ after the radix point. I give an extended definition of periodic numbers, and show the relationship between periodic and irrational numbers. I prove the transcendence of e by means of the factorial series and the factorial number system.
\end{abstract}

\section{Keywords}

Factorial Series, Factorial Number System, Periodic Numbers, Long Division, Periodic and Irrational Property, Transcendence of e

\section{Introduction}

Factorials have been known for thousands of years [1] [2]. They have been used in many fields of mathematics, including permutations, binomial coefficients, Bernoulli and Stirling numbers, central factorials [3], calculus, number theory, etc. Most literature on factorials focuses on integers rather than the non-integer part of the number.

The factorial number system [4] [5], though also known for centuries, is much less used in the study of irrational numbers than another tool, the theory of continued fractions [6]. Wikipedia deals with the factorial number system in 8 languages, while continued fractions are described in 42 languages. The reason for this discrepancy may be the great achievements of using continuous fractions by Bombelli, Wallis, Huygens, and especially by Lambert's proof of the irrationality of $\pi[7]$. 
When I started working on the factorial number system I felt that it was an unbiased system that could not be tied to a single, predefined number like binary or decimal number systems. This property was also present in continued fractions, but to me, the factorial system was more familiar, easier to work with, closer to known fixed-base systems than continued fractions. For me, using the factorial system was easier "to see through infinity" than other systems.

In this article, I focus on two main themes. One is the theory of periodic numbers in a new, extended definition, their properties and connections with rational and irrational numbers. The other topic concerns the proof of transcendence of e based on the factorial series and the factorial number system. In both cases, I followed simple, almost elementary, methods and techniques, including proving the transcendence of $\mathrm{e}$.

\section{Basic Features of the Factorial Number System}

\subsection{Defining the Distinction between the Factorial Series and the Factorial Number System}

In the present paper a real number $R$ is given in some form of a convergent factorial series

$$
R=\sum_{i=0}^{\infty}\left(r_{i} \frac{1}{i !}\right)=r_{0} \frac{1}{0 !}+r_{1} \frac{1}{1 !}+r_{2} \frac{1}{2 !}+\cdots+r_{i} \frac{1}{i !}+\cdots
$$

( $r_{i}$ is a non-negative integer $r_{i} \in Z^{0+}$ ).

Specifically, where indicated, I give the notation in the factorial number system. It is a special case of the factorial series:

$$
R=\sum_{i=0}^{\infty}\left(a_{i} \frac{1}{i !}\right)=a_{0} \frac{1}{0 !}+a_{1} \frac{1}{1 !}+a_{2} \frac{1}{2 !}+\cdots+a_{i} \frac{1}{i !}+\cdots
$$

where $a_{0}$ is any non-negative integer, $a_{1}$ is equal to 0 or 1 , and $0 \leq a_{i} \leq(i-1)$ for $i \geq 2$. The fractional part in this notation corresponds to the usual notation of a real number in the factorial number system. The reason for allowing any non-negative integer for $a_{0}$ is that I focus on the fractional part of the number in the present paper.

There are infinitely many ways to expand $R$ as the limit of a factorial series. In contrast, in the factorial number system there is only a single expansion when $R$ is irrational and two options when $R$ is rational.

\subsection{Examples of Expressing Rational and Irrational Numbers}

\section{1) Rational}

(in the factorial number system, $0 \leq a_{i} \leq(i-1)$ for $i \geq 1$ :)

$$
\begin{aligned}
& 01.000 \cdots=0 \frac{1}{0 !}+1 \frac{1}{1 !}+0 \frac{1}{2 !}+0 \frac{1}{3 !}+0 \frac{1}{4 !}+\cdots \\
& =00.123 \cdots=0 \frac{1}{0 !}+0 \frac{1}{1 !}+1 \frac{1}{2 !}+2 \frac{1}{3 !}+3 \frac{1}{4 !}+\cdots
\end{aligned}
$$

(in the factorial series, but not in the factorial number system:) 


$$
\begin{aligned}
& =0 \frac{1}{0 !}+0 \frac{1}{1 !}+(0 \times 2+1) \frac{1}{2 !}+0 \frac{1}{3 !}+(2 \times 4+3) \frac{1}{4 !}+0 \frac{1}{5 !}+(4 \times 6+5) \frac{1}{6 !}+\cdots \\
& =(0)(0) .(1)(0)(11)(0)(29)(0)+\cdots
\end{aligned}
$$

\section{2) Irrational}

(in the factorial number system, $0 \leq a_{i} \leq(i-1)$ for $i \geq 1$ )

$$
e=1 \frac{1}{0 !}+1 \frac{1}{1 !}+1 \frac{1}{2 !}+1 \frac{1}{3 !}+1 \frac{1}{4 !}+\cdots=(1)(1) .(1)(1)(1) \cdots
$$

(in the factorial series, but not in the factorial number system):

$$
\begin{aligned}
& =0 \frac{1}{0 !}+(1+1) \frac{1}{1 !}+0 \frac{1}{2 !}+(3+1) \frac{1}{3 !}+0 \frac{1}{4 !}+(5+1) \frac{1}{5 !}+(0) \frac{1}{6 !}+\cdots \\
& =(0)(2) \cdot(0)(4)(0)(6)(0)(8) \cdots \\
& =0 \frac{1}{0 !}+0 \frac{1}{1 !}+(1 \cdot 2+2+1) \frac{1}{2 !}+0 \frac{1}{3 !}+0 \frac{1}{4 !}+(4 \cdot 5+5+1) \frac{1}{5 !}+(0) \frac{1}{6 !}+(0) \frac{1}{7 !}+\ldots \\
& =(0)(0) \cdot(5)(0)(0)(26)(0)(0)(65) \cdots
\end{aligned}
$$

\subsection{Notation of Rational and Irrational Numbers in the Factorial Number System}

In the factorial number system all rational numbers have a terminating expansion with a last non-zero digit. The number of digits after the radix point to the last non-zero digit is less than or equal to the denominator of the given rational number, because there exists a factorial for any non-negative integer, and the denominator divides into its own factorial even if it does not divide into any smaller factorial [5].

There is also a non-terminating equivalent for every rational number which can be created by reducing the final non-zero term by 1 , then filling in the remaining infinite number of terms with the highest value possible for that position after the radix point.

$$
\begin{aligned}
& 01.000000 \cdots=00.123456789 \text { (10) (11) } \cdots \\
& 00.002000 \cdots=00.001456789 \text { (10) (11) } \cdots
\end{aligned}
$$

Just as with fixed-base systems, this ambiguity belongs to rational numbers only, while the fractional part of an irrational number admits one single expansion.

\subsection{Number Pictures}

For visual representation of the fractional part of a real number (deliberately neglecting the integer part here), it is of advantage to use number pictures based on the lattice points of the upper right octant of the coordinate system. Places after the radix point are displayed on the $x$-axis, and the corresponding digits on the $y$-axis. Lattice points on the $y=x$ line represent the maximal value allowed at the given place (Figure 1 ).

Example: $1 / 2 \mathrm{e}=00.01020304 \cdots$ (Figure 2):

\subsection{Arithmetic Operations in the Factorial Number System}

Addition and subtraction can be done on the usual pattern of fixed-base systems. The same applies for multiplication of a real number by a whole number. 


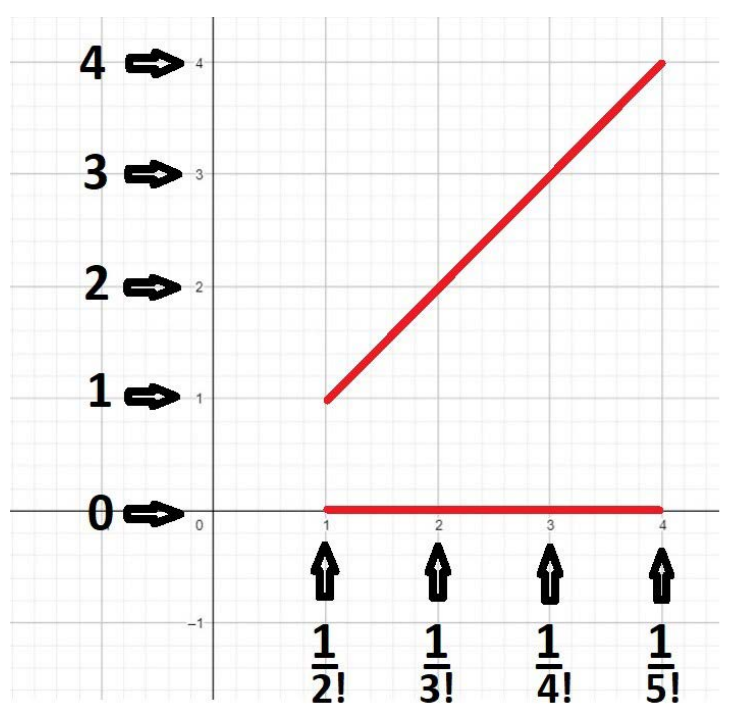

Figure 1. Lattice points of the number picture between the $x$-axis and the $y=x$ line.

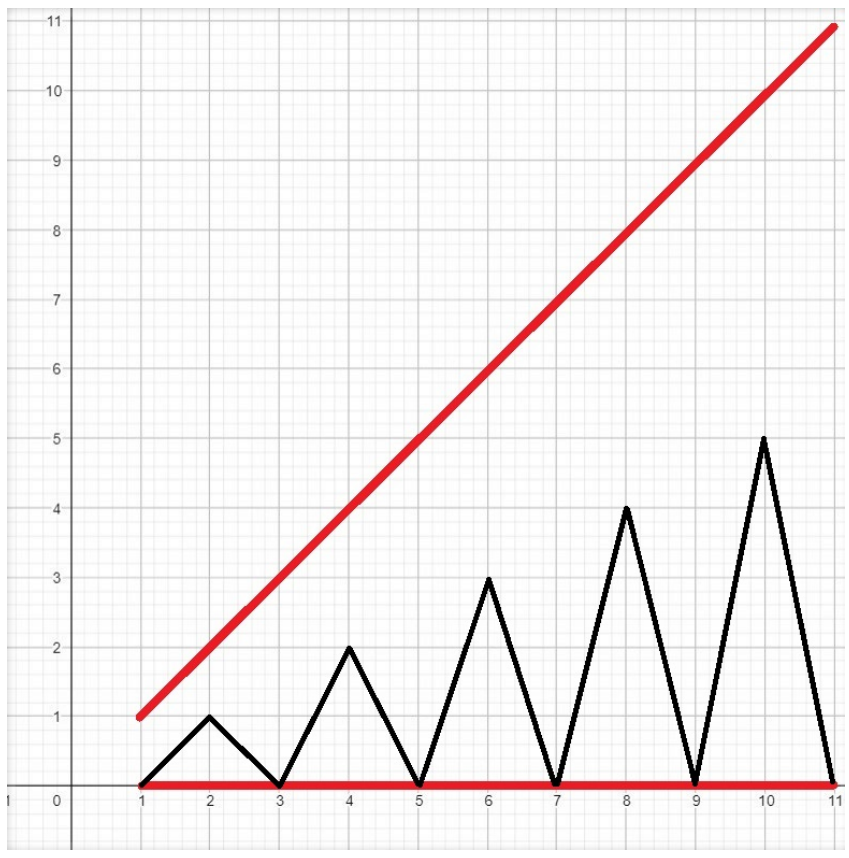

Figure 2. Number picture of $\frac{1}{2 \mathrm{e}}=00.01020304 \cdots$

Multiplication of two real numbers can be performed by using binomial coefficients and the diagonal method with sums along the southwest to northeast diagonals. This method works for any form of the two numbers in the factorial series, not only in the factorial number system. However, multiplying two real numbers given in the factorial number system does not necessarily yield the product in the proper form of the factorial number system, but only in the factorial series. Some digits may overflow the maximal value permitted at a given place, and the result has to be converted into the factorial number system.

Example: 00.1·00.1 (Table 1) 
Table 1. Multiplication in the factorial system.

\begin{tabular}{l|ccccc}
\hline & $0(1 / 0 !)$ & $0(1 / 1 !)$ & $1(1 / 2 !)$ & $0(1 / 3 !)$ & $0(1 / 4 !)$ \\
\hline $0(1 / 0 !)$ & $\left(\begin{array}{l}0 \\
0\end{array}\right) \cdot 0$ & $\left(\begin{array}{l}1 \\
0\end{array}\right) \cdot 0$ & $\left(\begin{array}{l}2 \\
0\end{array}\right) \cdot 0$ & $\left(\begin{array}{l}3 \\
0\end{array}\right) \cdot 0$ & $\left(\begin{array}{l}4 \\
0\end{array}\right) \cdot 0$ \\
$0(1 / 1 !)$ & $\left(\begin{array}{l}1 \\
1\end{array}\right) \cdot 0$ & $\left(\begin{array}{l}2 \\
1\end{array}\right) \cdot 0$ & $\left(\begin{array}{l}3 \\
1\end{array}\right) \cdot 0$ & $\left(\begin{array}{l}4 \\
1\end{array}\right) \cdot 0$ & $\left(\begin{array}{l}5 \\
1\end{array}\right) \cdot 0$ \\
$1(1 / 2 !)$ & $\left(\begin{array}{l}2 \\
2\end{array}\right) \cdot 0$ & $\left(\begin{array}{l}3 \\
2\end{array}\right) \cdot 0$ & $\left(\begin{array}{l}4 \\
2\end{array}\right) \cdot 1$ & $\left(\begin{array}{l}5 \\
2\end{array}\right) \cdot 0$ & $\left(\begin{array}{l}6 \\
2\end{array}\right) \cdot 0$ \\
$0(1 / 3 !)$ & $\left(\begin{array}{l}3 \\
3\end{array}\right) \cdot 0$ & $\left(\begin{array}{l}4 \\
3\end{array}\right) \cdot 0$ & $\left(\begin{array}{l}5 \\
3\end{array}\right) \cdot 0$ & $\left(\begin{array}{l}6 \\
3\end{array}\right) \cdot 0$ & $\left(\begin{array}{l}7 \\
3\end{array}\right) \cdot 0$ \\
$0(1 / 4 !)$ & $\left(\begin{array}{l}4 \\
4\end{array}\right) \cdot 0$ & $\left(\begin{array}{l}5 \\
4\end{array}\right) \cdot 0$ & $\left(\begin{array}{l}6 \\
4\end{array}\right) \cdot 0$ & $\left(\begin{array}{l}7 \\
4\end{array}\right) \cdot 0$ & $\left(\begin{array}{l}8 \\
4\end{array}\right) \cdot 0$ \\
\hline
\end{tabular}

$$
\begin{aligned}
00.1 \cdot 00.1= & {\left[\left(\left(\begin{array}{l}
0 \\
0
\end{array}\right) \cdot 0\right)\right] \frac{1}{0 !}+\left[\left(\begin{array}{l}
1 \\
1
\end{array}\right) \cdot 0+\left(\begin{array}{l}
1 \\
0
\end{array}\right) \cdot 0\right] \frac{1}{1 !} } \\
& +\left[\left(\begin{array}{l}
2 \\
2
\end{array}\right) \cdot 0+\left(\begin{array}{l}
2 \\
1
\end{array}\right) \cdot 0+\left(\begin{array}{l}
2 \\
0
\end{array}\right) \cdot 0\right] \frac{1}{2 !} \\
& +\left[\left(\begin{array}{l}
3 \\
3
\end{array}\right) \cdot 0+\left(\begin{array}{l}
3 \\
2
\end{array}\right) \cdot 0+\left(\begin{array}{l}
3 \\
1
\end{array}\right) \cdot 0+\left(\begin{array}{l}
3 \\
0
\end{array}\right) \cdot 0\right] \frac{1}{3 !} \\
& +\left[\left(\begin{array}{l}
4 \\
4
\end{array}\right) \cdot 0+\left(\begin{array}{l}
4 \\
3
\end{array}\right) \cdot 0+\left(\begin{array}{l}
4 \\
2
\end{array}\right) \cdot 1+\left(\begin{array}{l}
4 \\
1
\end{array}\right) \cdot 0+\left(\begin{array}{l}
4 \\
0
\end{array}\right) \cdot 0\right] \frac{1}{4 !} \\
= & (0)(0) \cdot(0)(0)(6)(0)(0) \cdots
\end{aligned}
$$

The result does not fit into the factorial number system, because it overflows at $\left(\begin{array}{l}4 \\ 2\end{array}\right) \cdot 1=6 \cdot \frac{1}{4 !}$, where the maximal value allowed is 3 . So we have to convert it further into the factorial number system:

$$
\begin{aligned}
0.1 \cdot 00.1 & =0 \cdot \frac{1}{0 !}+0 \cdot \frac{1}{1 !}+0 \cdot \frac{1}{2 !}+0 \cdot \frac{1}{3 !}+6 \cdot \frac{1}{4 !} \\
& =0 \cdot \frac{1}{0 !}+0 \cdot \frac{1}{1 !}+0 \cdot \frac{1}{2 !}+4 \cdot \frac{1}{4 !}+2 \cdot \frac{1}{4 !} \\
& =0 \cdot \frac{1}{0 !}+0 \cdot \frac{1}{1 !}+0 \cdot \frac{1}{2 !}+1 \cdot \frac{1}{3 !}+2 \cdot \frac{1}{4 !} \\
& =00.012
\end{aligned}
$$

\subsection{Division Algorithm of a Real Number by a Whole Number}

- In fixed-base systems: The base (e.g. $10,10,10, \ldots$ in the decimal system) is multiplied by the respective remainder.

- In the factorial number system: The algorithm is based on the series $1,1,2,3$, $4,5, \cdots$ to be multiplied by the respective remainder [8].

Example (Table 2): 
Table 2. Division of a rational number in the factorial number system.

\begin{tabular}{|c|c|}
\hline 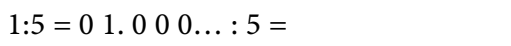 & $1: 5=00.12345 \ldots: 5=$ \\
\hline $01.000 \ldots: 5=00.010400000 \ldots$ & $\underline{00.12345 \ldots: 5}=00.0103567 \ldots$ \\
\hline$(0 \cdot 1): 5=0 \cdot 5+0$ & $(0 \cdot 1): 5=0 \cdot 5+0$ \\
\hline$(0 \cdot 1+1): 5=0 \cdot 5+1$ & $(0 \cdot 1+0): 5=0 \cdot 5+0$ \\
\hline$(1 \cdot 2+0): 5=0 \cdot 5+2$ & $(0 \cdot 2+1): 5=0 \cdot 5+1$ \\
\hline$(2 \cdot 3+0): 5=1 \cdot 5+1$ & $(1 \cdot 3+2): 5=1 \cdot 5+0$ \\
\hline$(1 \cdot 4+0): 5=0 \cdot 5+4$ & $(0 \cdot 4+3): 5=0 \cdot 5+3$ \\
\hline$(4 \cdot 5+0): 5=4 \cdot 5+0$ & $(3 \cdot 5+4): 5=3 \cdot 5+4$ \\
\hline$(0 \cdot 6+0): 5=0 \cdot 5+0$ & $(4 \cdot 6+5): 5=5 \cdot 5+4$ \\
\hline$(0 \cdot 7+0): 5=0 \cdot 5+0$ & $(4 \cdot 7+6): 5=6 \cdot 5+4$ \\
\hline$(0 \cdot 8+0): 5=0 \cdot 5+0$ & $(4 \cdot 8+7): 5=7 \cdot 5+4$ \\
\hline$\ldots$ & $\ldots$ \\
\hline
\end{tabular}

\section{Periodic Numbers}

1) In a fixed-base system, a periodic number contains a finite series of digits which is repeated infinitely. The same definition applies to continued fractions [6]. The following definition extends the concept of periodicity in the factorial number system. It is not a series of digits, but the differences between the corresponding digits of two consecutive series that are fixed.

Example: $\frac{\mathrm{e}}{3}$ (Table 3)

2) Definition: A real number $A=\left(a_{0}\right)\left(a_{1}\right)\left(a_{2}\right)\left(a_{3}\right) \cdots$ expanded in the factorial number system is periodic if there exists an index $r$, a natural number $N$, and non-negative integers $b_{r+1}, b_{r+2}, \cdots, b_{r+N}$ so that

$$
\begin{gathered}
a_{r+1 N+1}=a_{r+1}+1 b_{1} ; a_{r+1 N+2}=a_{r+2}+1 b_{2} ; \cdots ; a_{r+2 N}=a_{r+N}+1 b_{N} ; \\
a_{r+2 N+1}=a_{r+1}+2 b_{1} ; a_{r+2 N+2}=a_{r+2}+2 b_{2} ; \cdots ; a_{r+3 N}=a_{r+N}+2 b_{N} ; \\
\vdots \\
a_{r+k N+1}=a_{r+1}+k b_{1} ; a_{r+2 N+2}=a_{r+2}+k b_{2} ; \cdots ; a_{r+3 N}=a_{r+N}+k b_{N} ;
\end{gathered}
$$

The series of digits $\left(a_{0}\right)\left(a_{1}\right)\left(a_{2}\right)\left(a_{3}\right) \cdots\left(a_{r}\right)$ represents the introductory series,

$\left(a_{r+1}\right)\left(b_{a+2}\right) \cdots\left(a_{r+N}\right)$ the basis series;

$\left(b_{r+1}\right)\left(b_{r+2}\right) \cdots\left(b_{r+N}\right)$ the difference series of digits.

\section{3) Examples:}

$00.1000 \cdots$ : Introductory series: 00.1 ; basis series: 0 ; difference series: 0 .

$00.1000 \cdots=00.0234 \cdots$ : Introductory series: 00 0 0; basis series: 2 ; difference series: 1 .

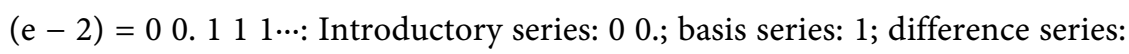
0 (Figure 3).

$\frac{\mathrm{e}}{3}=00.121342563 \cdots$ Introductory series: none; basis series: 0 0.1; difference series: 212 (Figure 4). 
Table 3. $\frac{\mathrm{e}}{3}=00.1|213| 425|637| \cdots$ (difference series 212 ).

\begin{tabular}{ccccccc}
\hline 0 & 0. & 1 & $=$ & 0 & 0. & 1 \\
2 & 1 & 3 & $=$ & $(0+2)$ & $(0+1)$ & $(1+2)$ \\
4 & 2 & 5 & $=$ & $(2+2)$ & $(1+1)$ & $(3+2)$ \\
6 & 3 & $7 \ldots$ & $=$ & $(4+2)$ & $(2+1)$ & $(5+2) \ldots$ \\
\hline
\end{tabular}

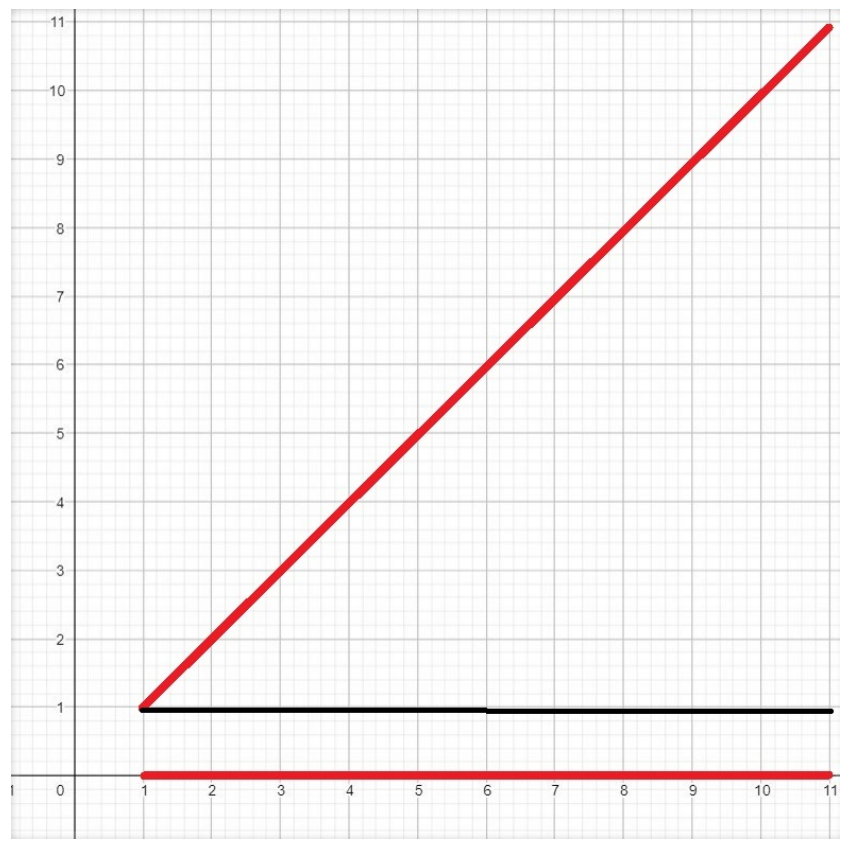

Figure 3. Number picture of $(\mathrm{e}-2)=00.1111 \cdots$.

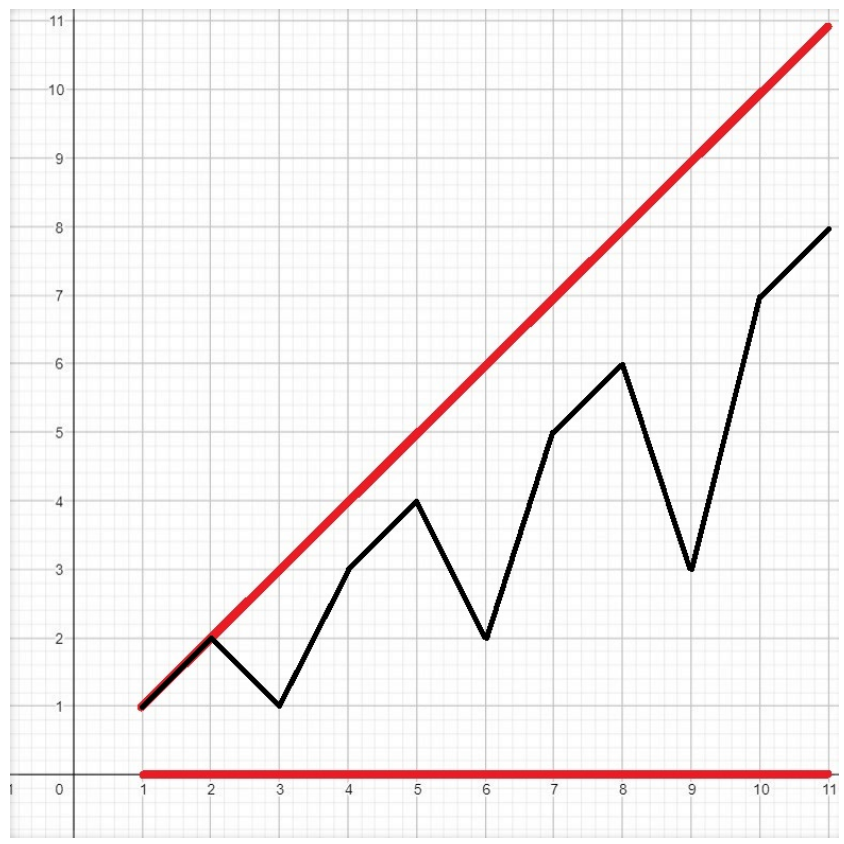

Figure 4. Number picture of $\frac{\mathrm{e}}{3}=00.1213425637 \cdots$. 


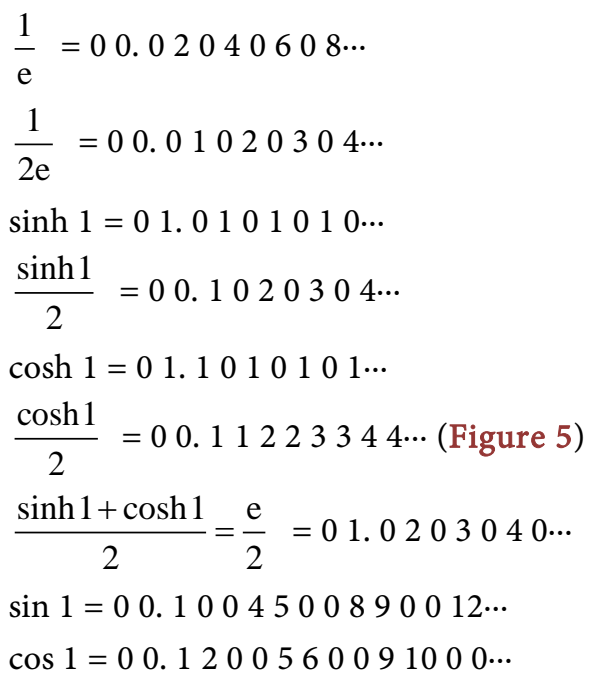

4) Create periodic numbers by the factorial division algorithm

New periodic numbers can be created from a given one by the factorial division algorithm (Table 4).

Table 4. $\frac{\mathrm{e}}{6}$ generated by the factorial division algorithm.

\begin{tabular}{lll}
\hline$(1 \cdot 1) / 6=0$ & $\frac{(2 \cdot 6+1)}{(1 \cdot 7+6=2 \cdot 6+1}$ & $\underline{(2 \cdot 12+1)}: 6=4 \cdot 6+1$ \\
$(1 \cdot 1+1): 6=0 \cdot 6+2$ & $\frac{(1 \cdot 6=1 \cdot 6+2}{(2 \cdot 8+1)}: 6=2 \cdot 6+5$ & $\underline{(1 \cdot 13+1)}: 6=2 \cdot 6+2$ \\
$(2 \cdot 2+1): 6=0 \cdot 6+5$ & $\underline{(2 \cdot 14+1)}: 6=4 \cdot 6+5$ \\
$(5 \cdot 3+1): 6=2 \cdot 6+4$ & $\underline{(5 \cdot 9+1)}: \frac{6=7 \cdot 6+4}{(5 \cdot 10+1)}: 6=6 \cdot 6+5$ & $\underline{(5 \cdot 15+1)}: 6=12 \cdot 6+4$ \\
$(4 \cdot 4+1): 6=2 \cdot 6+5$ & $\underline{(5 \cdot 11+1)}: \underline{6=9 \cdot 6+2}$ & $\underline{(5 \cdot 17+1)}: \underline{6=14 \cdot 6+2}$ \\
$(5 \cdot 5+1): 6=4 \cdot 6+2$ &
\end{tabular}

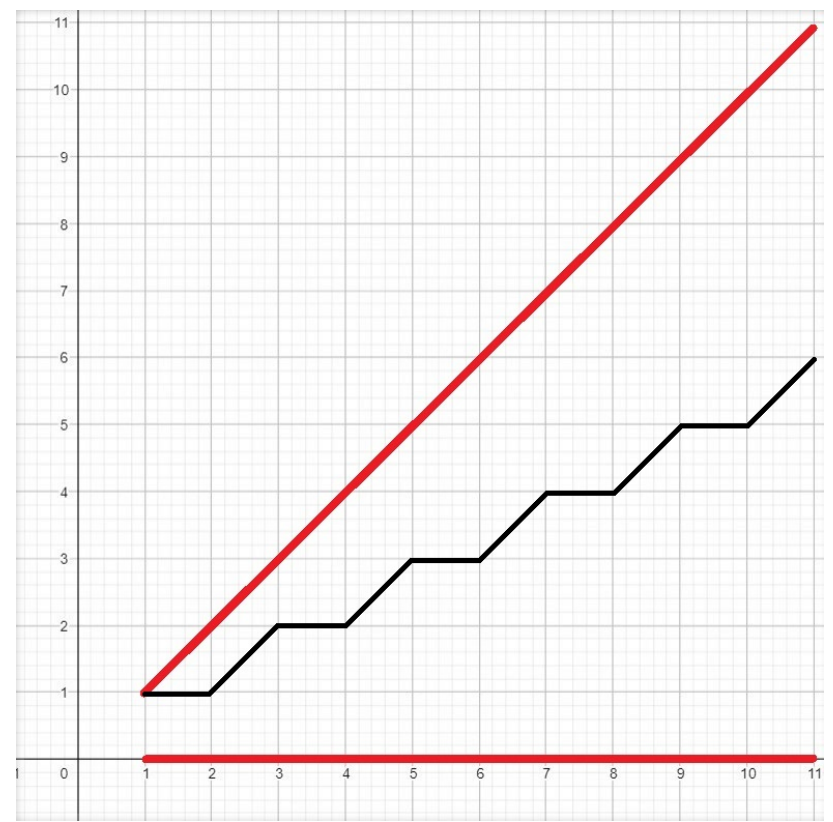

Figure 5. Number picture of $\frac{\cosh 1}{2}=00.11223344 \cdots$. 


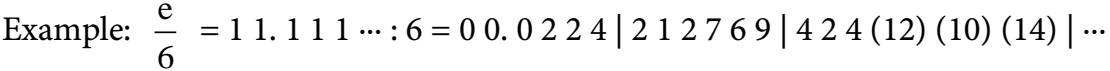

$$
\begin{aligned}
& =\underline{11.111 \cdots: 6}=00.0224|212769| 424 \text { (12) (10) (14) } \mid \cdots
\end{aligned}
$$

Introductory series: none; basis series: 00.022 4; difference series: 212545 (Figure 6).

\section{Theorems about Periodic Numbers}

I give a detailed proof for Theorems 3.7 and 3.8.

Theorem 3.1. From the definition it follows that the two types of expansion of rational numbers are periodic with length of period " 1 ". All other periodic numbers are irrational, including those with length of period " 1 " after an introductory series (for example $3-\mathrm{e}=0 \begin{array}{lllllllll}0 & 0 & 1 & 2 & 3 & \cdots\end{array}$ ), or those in which the minimal value $r_{1}=0$ and the maximal value $r_{1}=(i-1)$ at $r_{1} \frac{1}{i !}$ are oscillating, as in $\cos 1=00.120056009$ (10) … (Figure 7).

Theorem 3.2. The set of periodic numbers with respect to addition is an Abelian group with $00.000 \cdots$ as the unit element.

Theorem 3.3. The product of two periodic numbers may or may not be a periodic number. For example, e is periodic, as is the product of e with any rational number, but $\mathrm{e}^{2}$ is not periodic in the factorial number system.

Theorem 3.4. If a number is periodic after an introductory series of length $M$, it is also periodic after an introductory series of length $M^{\prime} \geq M$.

Theorem 3.5. If a number is periodic by length of period $N$, it is also periodic by $2 N, 3 N, \cdots$ Conversely, for every periodic number there exists a natural number

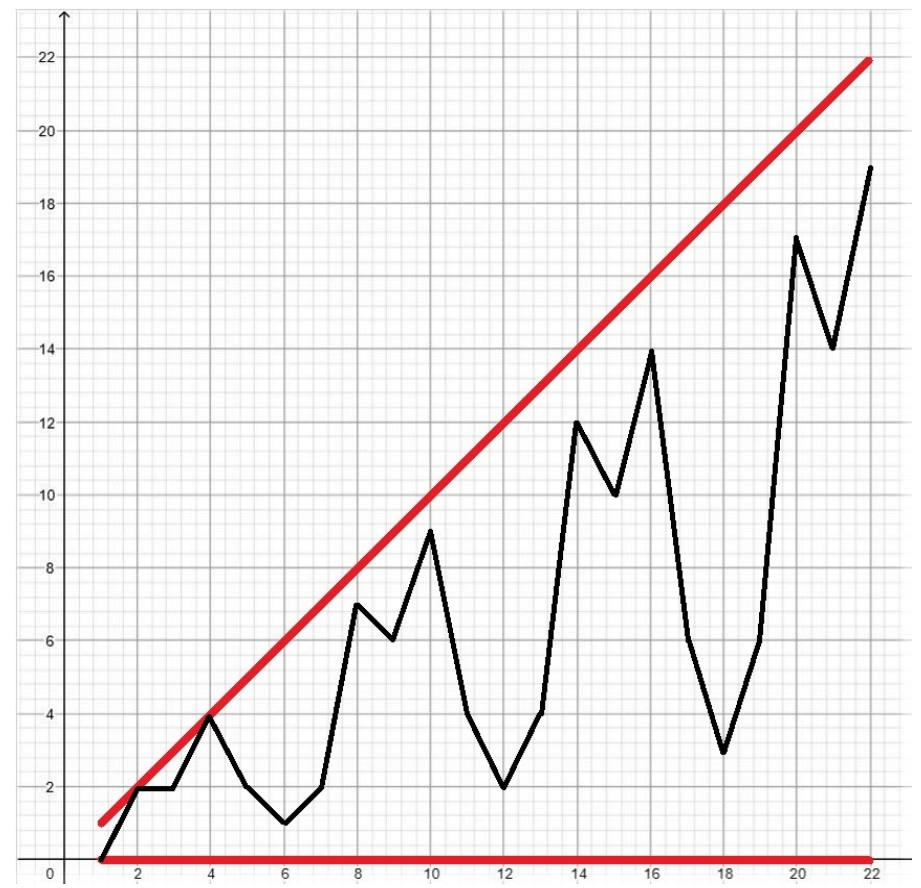

Figure 6. Number picture of $\frac{\mathrm{e}}{6}=00.0224|212769| 424$ (12) (10) (14)|*. 


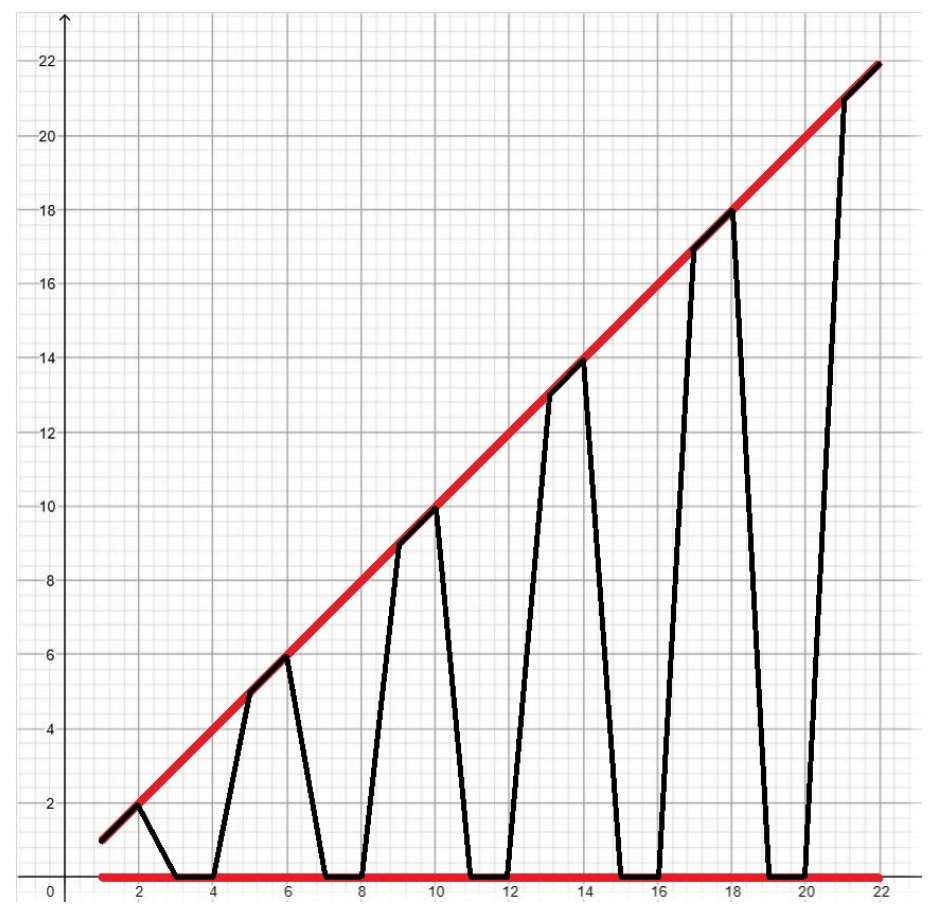

Figure 7. Number picture of $\cos 1=00.120056009$ (10) $\cdots$.

$N$ as the minimal length of the period. For example, $00.11223344 \cdots$ is periodic by 2 , but also by $2 n$ where $n$ is any natural number.

Theorem 3.6. In a periodic number with length of period $N$, no element in the difference series can be greater than $N$, because in this case there would be a digit in the number which is greater than the maximum allowed at the given index.

Theorem 3.7. If a number is periodic by $N$ and $N^{*}$ where $N$ and $N^{*}$ are co-primes (relative primes), this number is periodic by 1 .

The proof is cumbersome to describe in the general case. I prove the theorem for the special case $N=2$ and $N^{*}=3$ which shows the principle of proof for any two co-primes.

Given a periodic number of period 2 which means that after an introductory series the digits are $(a+1 c)(b+1 d)|(a+2 c)(b+2 d)|(a+3 c)(b+3 d) \mid(a+$ $4 c)(b+4 d) \mid(a+5 c)(b+5 d) \cdots$ where the parentheses indicate the consecutive digits of the number (not multiplication). The basis series is $a b$, and the difference series $c d$.

Suppose that this number is also periodic of period 3 after the same introductory series:

$(a+1 c)(b+1 d)(a+2 c)|(b+2 d)(a+3 c)(b+3 d)|(a+4 c)(b+4 d)(a+5 c)$ $\mid(b+5 d) \cdots$

This means that the basis series of period 3 is $(a+1 c)(b+1 d)(a+2 c)$, and the stair series can be calculated from the differences at the respective places (non-bold parentheses are in the ordinary sense of calculation):

$$
(b+2 d)-(a+1 c)=(a+4 c)-(b+2 d)
$$




$$
\begin{gathered}
(b+5 d)-(a+4 c)=(a+4 c)-(b+2 d) \\
3 d=3 c \text { and } c=d .
\end{gathered}
$$

This gives that the only solution for the elements $c$ and $d$ of the difference series is the same digit, but Theorem 6 gives that this digit can only be equal to 0 or 1. Q.E.D.

Theorem 3.8. The set of periodic numbers in the $[0,1)$ interval is countable.

Faber [9] proved the countable property of rational numbers by using the factorial expansion. In like manner, we take all periodic numbers with length of 1 digit. The introductory series to a given index $i$ can be chosen in 1 ? different ways, so this is a countable set. The basis series of length 1 can be any natural number, the step period either 0 or 1 , so this is also a countable set. For length of 2 digits, the introductory series is the same as with length 1, the basis series can be any pair of natural numbers, and the difference series can be chosen in $3^{2}$ different ways $(00,01,10,11,02,20,12,21,22)$, so this is also a countable set. In this manner, each series determines a countable set of periodic numbers. A countable union of countable sets is countable, so the set of periodic numbers in the [0, 1) interval is countable.

\section{Double factorials}

The odd and even double factorials generate two different number systems. The even factorial system is in many ways similar to the simple factorial system. It expresses all rational numbers in a finite form, and allows for periodic numbers, just like in the factorial system. The odd factorial system also admits periodic numbers, but an even-denominator fraction is expanded into an infinite periodic number.

\section{Extending the Definition of Periodicity in the Factorial Series}

1) Following is an extension of periodicity from the factorial number system to the factorial series so that the length of a period remains constant.

In the factorial number system there is a constant difference series between two finite consecutive series of digits (see 2.1.)

Extend this definition so that it is not necessarily the difference between the two corresponding digits, but a well-defined function $f(k)$ that expresses the variance of $r_{k}$ according to $f(k)$ at a given place after the radix point. Therefore, it is appropriate in these cases to use the expression "variance series" rather than "difference series".

It follows from the broader definition that the periodic numbers in the factorial number system retain this property by the new definition.

Extending the concept of periodicity in this way, many noteworthy numbers that are not periodic in the factorial number system, can be expressed in periodic form in the factorial series. 
2) Examples:

$$
\begin{aligned}
& \infty=0 ! \frac{1}{0 !}+1 ! \frac{1}{1 !}+2 ! \frac{1}{2 !}+3 ! \frac{1}{3 !}+\cdots=(0)(1 !) \cdot(2 !)(3 !) \cdots \\
& =\sum_{k=0}^{\infty} k ! \frac{1}{k !}=1+1+1+\cdots \\
& \infty=0 ! \frac{1}{1 !}+1 ! \frac{1}{2 !}+2 ! \frac{1}{3 !}+3 ! \frac{1}{4 !}+\cdots=(0)(0 !) .(1 !)(2 !) \cdots \\
& =\sum_{k=0}^{\infty} k ! \frac{1}{(k+1) !}=\frac{1}{1}+\frac{1}{2}+\frac{1}{3}+\cdots \\
& 1=0 ! \frac{1}{2 !}+1 ! \frac{1}{3 !}+2 ! \frac{1}{4 !}+3 ! \frac{1}{5 !}(0)(0 !) \cdot(0 !)(1 !)(2 !) \cdots \\
& =\sum_{k=0}^{\infty} k ! \frac{1}{(k+2) !}=\frac{1}{1 \cdot 2}+\frac{1}{2 \cdot 3}+\cdots \\
& \mathrm{e}^{1}=1^{0} \frac{1}{0 !}+1^{1} \frac{1}{1 !}+1^{2} \frac{1}{2 !}+1^{3} \frac{1}{3 !}+1^{4} \frac{1}{4 !}+\cdots=(1)(1) \cdot(1)(1)(1) \cdots \\
& \mathrm{e}^{2}=2^{0} \frac{1}{0 !}+2^{1} \frac{1}{1 !}+2^{2} \frac{1}{2 !}+2^{3} \frac{1}{3 !}+\cdots=\left(2^{0}\right)\left(2^{1}\right) \cdot\left(2^{2}\right)\left(2^{3}\right)\left(2^{4}\right) \cdots \\
& \mathrm{e}^{n}=n^{0} \frac{1}{0 !}+n^{1} \frac{1}{1 !}+n^{2} \frac{1}{2 !}+n^{3} \frac{1}{3 !}+n^{4} \frac{1}{4 !}+\cdots=\left(n^{0}\right)\left(n^{1}\right) \cdot\left(n^{2}\right)\left(n^{3}\right)\left(n^{4}\right) \cdots \\
& \ln 2=\frac{1}{1}-\frac{1}{2}+\frac{1}{3}-\frac{1}{4}+\frac{1}{5}-\frac{1}{6}+\frac{1}{7}-\frac{1}{8}+\cdots=\frac{1}{1 \cdot 2}+\frac{1}{3 \cdot 4}+\frac{1}{5 \cdot 6}+\cdots \\
& =(0) \frac{1}{0 !}+(0) \frac{1}{1 !}+(0 !) \frac{1}{2 !}+(0) \frac{1}{3 !}+(2 !) \frac{1}{4 !} \\
& +(0) \frac{1}{5 !}+(4 !) \frac{1}{6 !}+(0) \frac{1}{7 !}+(6 !) \frac{1}{8 !}+\cdots \\
& =\sum_{k=1}^{\infty}[(2 k-2) !] \frac{1}{(2 k) !}=(0)(0) \cdot(0 !)(0)(2 !)(0)(4 !)(0)(6 !)(0)(8 !) \cdots \\
& \frac{\pi}{8}=\frac{1}{2}\left(\frac{1}{1}-\frac{1}{3}+\frac{1}{5}-\frac{1}{7}+\frac{1}{9}-\frac{1}{11}+\frac{1}{13}-\frac{1}{15}+\cdots\right) \\
& =0 \frac{1}{0 !}+0 \frac{1}{1 !}+0 \frac{1}{2 !}+0 ! \cdot 2 \frac{1}{3 !}+0 \frac{1}{4 !}+0 \frac{1}{5 !}+0 \frac{1}{6 !}+4 ! \cdot 6 \frac{1}{7 !}+0 \frac{1}{8 !} \\
& +0 \frac{1}{9 !}+0 \frac{1}{10 !}+8 ! \cdot 10 \frac{1}{11 !}+\cdots \\
& =\sum_{k=1}^{\infty}[(4 k-4) !(4 k-2)] \frac{1}{(4 k-1) !} \\
& =(0)(0) \cdot(0)(0 ! \cdot 2)(0)(0)(0)(4 ! \cdot 6)(0)(0)(0)(8 ! \cdot 10)(0)(0)(0)(12 ! \cdot 14) \cdots \\
& =\left(0 ! \frac{1}{3 !}+4 ! \frac{1}{7 !}+8 ! \frac{1}{11 !}+\cdots\right)+\left(1 ! \frac{1}{3 !}+5 ! \frac{1}{7 !}+9 ! \frac{1}{11 !}+\cdots\right) \\
& =(0)(0) \cdot(0)(0 !)(0)(0)(0)(4 !)(0)(0)(0)(8 !) \cdots \\
& +(0)(0) \cdot(0)(1 !)(0)(0)(0)(5 !)(0)(0)(0)(9 !) \cdots
\end{aligned}
$$

Riemann numbers:

For example, the Apéry number: 


$$
\begin{aligned}
\zeta(3) & =\sum_{k=0}^{\infty} \frac{(3 k) !}{k^{3}} \frac{1}{(3 k) !}=\sum_{k=0}^{\infty}(3 !) \frac{(k-1) !(2 k-1) !(3 k-1) !}{(0 k) !(1 k) !(2 k) !} \frac{1}{(3 k) !} \\
\frac{\zeta(3)}{3 !} & =(0)(0) \cdot(0)\left(\frac{0 ! 1 ! 2 !}{0 ! 1 ! 2 !}\right)(0)(0)\left(\frac{1 ! 3 ! 5 !}{0 ! 2 ! 4 !}\right)(0)(0)\left(\frac{2 ! 5 ! 8 !}{0 ! 3 ! 6 !}\right) \cdots \\
& =\sum_{k=0}^{\infty} \frac{(k-1) !(2 k-1) !(3 k-1) !}{(0 k) !(1 k) !(2 k) !} \frac{1}{(3 k) !}
\end{aligned}
$$

\section{7. e Transcendental}

1) Here I consider expansions not only in the factorial number system, but other expansions in the factorial series, where some $r_{i}$ digits may overflow the upper limit $(i-1)$ at the given place.

$$
\begin{aligned}
& \mathrm{e}^{n}=\sum_{k=0}^{\infty}\left(n^{k} \frac{1}{k !}\right)=n^{0} \frac{1}{0 !}+n^{1} \frac{1}{1 !}+n^{2} \frac{1}{2 !}+\cdots+n^{k} \frac{1}{k !}+\cdots \\
& \text { Suppose } c_{0} \mathrm{e}^{0}+c_{1} \mathrm{e}^{1}+c_{2} \mathrm{e}^{2}+\cdots+c_{i} \mathrm{e}^{i}+\cdots+c_{n-1} \mathrm{e}^{n-1}+c_{n} \mathrm{e}^{n}=0
\end{aligned}
$$

( $n$ fixed natural number, $c_{i}$ integer). I prove that the only solution for all $c_{i}$ coefficients is the trivial $c_{i}=0$.

I give the proof for $n=3$, and generalize for arbitrary $n$.

$$
\text { Suppose } c_{0} \mathrm{e}^{0}+c_{1} \mathrm{e}^{1}+c_{2} \mathrm{e}^{2}+c_{3} \mathrm{e}^{3}=0 \quad\left(c_{i} \text { integer }\right) \text {. }
$$

All of the series $c_{0} \mathrm{e}^{0}, c_{1} \mathrm{e}^{1}, c_{2} \mathrm{e}^{2}, c_{3} \mathrm{e}^{3}$ have absolute convergence, so they can be rearranged in the following way:

$$
\begin{aligned}
-c_{0} \mathrm{e}^{0}= & c_{1} \mathrm{e}^{1}+c_{2} \mathrm{e}^{2}+c_{3} \mathrm{e}^{3} \\
= & c_{1} 1^{0} \frac{1}{0 !}+c_{1} 1^{1} \frac{1}{1 !}+c_{2} 1^{2} \frac{1}{2 !}+\cdots+c_{1} 1^{k} \frac{1}{k !}+\cdots \\
& +c_{1} 2^{0} \frac{1}{0 !}+c_{1} 2^{1} \frac{1}{1 !}+c_{2} 2^{2} \frac{1}{2 !}+\cdots+c_{1} 2^{k} \frac{1}{k !}+\cdots \\
& +c_{1} 3^{0} \frac{1}{0 !}+c_{1} 3^{1} \frac{1}{1 !}+c_{2} 3^{2} \frac{1}{2 !}+\cdots+c_{1} 3^{k} \frac{1}{k !}+\cdots \\
= & \left(c_{1} 1^{0}+c_{2} 2^{0}+c_{3} 3^{0}\right) \frac{1}{0 !}+\left(c_{1} 1^{1}+c_{2} 2^{1}+c_{3} 3^{1}\right) \frac{1}{1 !} \\
& +\left(c_{1} 1^{2}+c_{2} 2^{2}+c_{3} 3^{2}\right) \frac{1}{2 !}+\cdots+\left(c_{1} 1^{k}+c_{2} 2^{k}+c_{3} 3^{k}\right) \frac{1}{k !}
\end{aligned}
$$

Since $-c_{0} \mathrm{e}^{0}=c_{1} \mathrm{e}^{1}+c_{2} \mathrm{e}^{2}+c_{3} \mathrm{e}^{3}$ is an integer, it has an extension in the factorial number system where all $r_{k}$ coefficients of $\frac{1}{k !}$ after the radix point, from $\frac{1}{2 !}$ are equal to zero $(2 \leq k<\infty)$ :

$$
\begin{gathered}
c_{1} 1^{2}+c_{2} 2^{2}+c_{3} 3^{2}=0 \\
c_{1} 1^{3}+c_{2} 2^{3}+c_{3} 3^{3}=0 \\
c_{1} 1^{4}+c_{2} 2^{4}+c_{3} 3^{4}=0 \\
\vdots \\
c_{1} 1^{k}+c_{2} 2^{k}+c_{3} 3^{k}=0
\end{gathered}
$$

Consider the first three equations: 


$$
\begin{aligned}
& c_{1} 1^{2}+c_{2} 2^{2}+c_{3} 3^{2}=0 \\
& c_{1} 1^{3}+c_{2} 2^{3}+c_{3} 3^{3}=0 \\
& c_{1} 1^{4}+c_{2} 2^{4}+c_{3} 3^{4}=0
\end{aligned}
$$

This is a homogeneous system of linear equations with $c_{1}, c_{2}, c_{3}$ unknowns.

If there is any solution different from the trivial $c_{i}=0$, the determinant of the coefficients must be equal to zero:

$$
D_{3}=\left|\begin{array}{ccc}
1^{2} & 2^{2} & 3^{2} \\
1^{3} & 2^{3} & 3^{3} \\
1^{4} & 2^{4} & 3^{4}
\end{array}\right|=1^{2} 2^{2} 3^{2} \cdot\left|\begin{array}{ccc}
1^{0} & 2^{0} & 3^{0} \\
1^{1} & 2^{1} & 3^{1} \\
1^{2} & 2^{2} & 3^{2}
\end{array}\right|=\left(1^{2} 2^{2} 3^{2}\right) \cdot V_{3}
$$

This is a Vandermonde determinant with 3 different base numbers, multiplied by $1^{2} 2^{2} 3^{2}$. Its numerical value differs from zero, so the only solution for $c_{1}, c_{2}, c_{3}$ unknowns is the trivial one: $c_{1}=c_{2}=c_{3}=0$.

Generalize for arbitrary fixed $n$ :

$$
\begin{aligned}
& \mathrm{e}^{n}=\sum_{k=0}^{\infty}\left(n^{k} \frac{1}{k !}\right)=n^{0} \frac{1}{0 !}+n^{1} \frac{1}{1 !}+n^{2} \frac{1}{2 !}+\cdots+n^{k} \frac{1}{k !}+\cdots \\
& c_{0} \mathrm{e}^{0}+c_{1} \mathrm{e}^{1}+c_{2} \mathrm{e}^{2}+c_{3} \mathrm{e}^{3}+\cdots+c_{i} \mathrm{e}^{i}+\cdots+c_{n-1} \mathrm{e}^{n-1}+c_{n} \mathrm{e}^{n}=0
\end{aligned}
$$

All of these series have absolute convergence, so they can be rearranged in the following way:

$$
\begin{aligned}
-c_{0} \mathrm{e}^{0}= & c_{1} \mathrm{e}^{1}+c_{2} \mathrm{e}^{2}+c_{3} \mathrm{e}^{3}+\cdots+c_{i} \mathrm{e}^{i}+\cdots+c_{n-1} \mathrm{e}^{n-1}+c_{n} \mathrm{e}^{n} \\
= & c_{1} 1^{0} \frac{1}{0 !}+c_{1} 1^{1} \frac{1}{1 !}+c_{2} 1^{2} \frac{1}{2 !}+\cdots+c_{1} 1^{k} \frac{1}{k !}+\cdots \\
& +c_{1} 2^{0} \frac{1}{0 !}+c_{1} 2^{1} \frac{1}{1 !}+c_{2} 2^{2} \frac{1}{2 !}+\cdots+c_{1} 2^{k} \frac{1}{k !}+\cdots \\
& +c_{1} 3^{0} \frac{1}{0 !}+c_{1} 3^{1} \frac{1}{1 !}+c_{2} 3^{2} \frac{1}{2 !}+\cdots+c_{1} 3^{k} \frac{1}{k !}+\cdots \\
& +\cdots \\
& +c_{1} n^{0} \frac{1}{0 !}+c_{1} n^{1} \frac{1}{1 !}+c_{2} n^{2} \frac{1}{2 !}+\cdots+c_{1} n^{k} \frac{1}{k !}+\cdots \\
= & \left(c_{1} 1^{0}+c_{2} 2^{0}+c_{3} 3^{0}+\cdots+c_{n} n^{0}\right) \frac{1}{0 !} \\
& +\left(c_{1} 1^{1}+c_{2} 2^{1}+c_{3} 3^{1}+\cdots+c_{n} n^{1}\right) \frac{1}{1 !} \\
& +\left(c_{1} 1^{2}+c_{2} 2^{2}+c_{3} 3^{2}+\cdots+c_{n} n^{2}\right) \frac{1}{2 !} \\
& +\cdots \\
& +\left(c_{1} 1^{k}+c_{2} 2^{k}+c_{3} 3^{k}+\cdots+c_{n} n^{k}\right) \frac{1}{k !}
\end{aligned}
$$

Since $-c_{0} \mathrm{e}^{0}=c_{1} \mathrm{e}^{1}+c_{2} \mathrm{e}^{2}+c_{3} \mathrm{e}^{3}+\cdots+c_{i} \mathrm{e}^{i}+\cdots+c_{n-1} \mathrm{e}^{n-1}+c_{n} \mathrm{e}^{n}$ is an integer, it has an extension in the factorial number system where all $r_{k}$ coefficients of $\frac{1}{k !}$ after the radix point, from $\frac{1}{2 !}$ are equal to zero $(2 \leq k<\infty)$ : 


$$
\begin{gathered}
c_{1} 1^{2}+c_{2} 2^{2}+c_{3} 3^{2}+\cdots+c_{n} n^{2}=0 \\
c_{1} 1^{3}+c_{2} 2^{3}+c_{3} 3^{3}+\cdots+c_{n} n^{3}=0 \\
c_{1} 1^{4}+c_{2} 2^{4}+c_{3} 3^{4}+\cdots+c_{n} n^{4}=0 \\
\vdots \\
c_{1} 1^{k}+c_{2} 2^{k}+c_{3} 3^{k}+\cdots+c_{n} n^{k}=0
\end{gathered}
$$

Consider the first $n$ equations:

$$
\begin{gathered}
c_{1} 1^{2}+c_{2} 2^{2}+c_{3} 3^{2}+\cdots+c_{n} n^{2}=0 \\
c_{1} 1^{3}+c_{2} 2^{3}+c_{3} 3^{3}+\cdots+c_{n} n^{3}=0 \\
c_{1} 1^{4}+c_{2} 2^{4}+c_{3} 3^{4}+\cdots+c_{n} n^{4}=0 \\
\vdots \\
c_{1} 1^{n+1}+c_{2} 2^{n+1}+c_{3} 3^{n+1}+\cdots+c_{n} n^{n+1}=0
\end{gathered}
$$

This is a homogeneous system of linear equations with $c_{1}, c_{2}, c_{3}, \cdots, c_{n}$ unknowns. If there is any solution different from $c_{i}=0$, the determinant of the coefficients must be equal to zero. However, the determinant can be transformed into a Vandermonde determinant multiplied by a constant, in the same way as for $n=3$.

$$
D_{n}=\left|\begin{array}{ccc}
1^{2} & \cdots & n^{2} \\
\vdots & \ddots & \vdots \\
1^{n+1} & \cdots & n^{n+1}
\end{array}\right|=\left(1^{2} 2^{2} 3^{2} \cdots n^{2}\right) \cdot\left|\begin{array}{ccc}
1^{0} & \cdots & n^{0} \\
\vdots & \ddots & \vdots \\
1^{n-1} & \cdots & n^{n-1}
\end{array}\right|=\left(1^{2} 2^{2} 3^{2} \cdots n^{2}\right) \cdot V_{n}
$$

This is a Vandermonde determinant with $n$ different base numbers multiplied by $1^{2} 2^{2} 3^{2} \cdots n^{2}$. Its numerical value differs from zero for any fixed $n$. The only solution for all $c_{i}$ unknowns is the trivial one: $c_{i}=0$. Therefore, e is transcendental. Q. E. D.

Corollary 1. All integer powers of e are irrational numbers.

Corollary 2. $\ln 2$ is irrational.

\section{2) The infinite form of $e$}

Any rational number has two different expansions in the factorial system, as in fixed-base systems. The above proof is based on the finite form that consists of zero digits after the radix point.

However, any rational number has two possible extensions in the factorial number system. Therefore the non-existence of the infinite form follows from the above proof of the non-existence of the finite form.

This means that, if $\left(-c_{0} \mathrm{e}^{0}\right)$ is an integer, it has another expansion in the factorial number system in which all digits after the radix point have maximal value allowed at the given place:

$$
\begin{gathered}
c_{1} 1^{2}+c_{2} 2^{2}+c_{3} 3^{2}+\cdots+c_{n} n^{2}=1 \\
c_{1} 1^{3}+c_{2} 2^{3}+c_{3} 3^{3}+\cdots+c_{n} n^{3}=2 \\
c_{1} 1^{4}+c_{2} 2^{4}+c_{3} 3^{4}+\cdots+c_{n} n^{4}=3 \\
\vdots \\
c_{1} 1^{k}+c_{2} 2^{k}+c_{3} 3^{k}+\cdots+c_{n} n^{k}=(k-1)
\end{gathered}
$$


This is an infinitely many times over-determined system of equations for $n$ unknowns $c_{1}, c_{2}, c_{3}, \cdots, c_{n}$. From the above proof about the non-existence of the finite form it follows that solving the first $n$ equations, and substituting $c_{1}, c_{2}, c_{3}, \cdots, c_{n}$ into any one of the remaining equations, the system of $n+1$ equations is always inconsistent.

Consider the first $n$ equations:

$$
\begin{gathered}
c_{1} 1^{2}+c_{2} 2^{2}+c_{3} 3^{2}+\cdots+c_{n} n^{2}=1 \\
c_{1} 1^{3}+c_{2} 2^{3}+c_{3} 3^{3}+\cdots+c_{n} n^{3}=2 \\
c_{1} 1^{4}+c_{2} 2^{4}+c_{3} 3^{4}+\cdots+c_{n} n^{4}=3 \\
\vdots \\
c_{1} 1^{n+1}+c_{2} 2^{n+1}+c_{3} 3^{n+1}+\cdots+c_{n} n^{n+1}=n
\end{gathered}
$$

Apart from the indirect reasoning via the finite form above, this statement can also be justified by direct proof. Following is a proof for $n=3$ that can be generalized for arbitrary $n$.

Given the first three equations:

$$
\begin{aligned}
& c_{1} 1^{2}+c_{2} 2^{2}+c_{3} 3^{2}=1 \\
& c_{1} 1^{3}+c_{2} 2^{3}+c_{3} 3^{3}=2 \\
& c_{1} 1^{4}+c_{2} 2^{4}+c_{3} 3^{4}=3
\end{aligned}
$$

Solving this system of equations for, say, $c_{3}$ with Cramer's rule:

$$
c_{3}=\frac{\left|\begin{array}{lll}
1^{2} & 2^{2} & 1 \\
1^{3} & 2^{3} & 2 \\
1^{4} & 2^{4} & 3
\end{array}\right|}{\left|\begin{array}{lll}
1^{2} & 2^{2} & 3^{2} \\
1^{3} & 2^{3} & 3^{3} \\
1^{4} & 2^{4} & 3^{4}
\end{array}\right|}=\frac{1^{2} 2^{2}}{1^{2} 2^{2} 3^{2}} \frac{\left|\begin{array}{ccc}
1^{0} & 2^{0} & 1 \\
1^{1} & 2^{1} & 2 \\
1^{2} & 2^{2} & 3
\end{array}\right|}{\left|\begin{array}{ccc}
1^{0} & 2^{0} & 3^{0} \\
1^{1} & 2^{1} & 3^{1} \\
1^{2} & 2^{2} & 3^{2}
\end{array}\right|}
$$

Now consider another three equations ( $k \geq 0, k$ integer):

$$
\begin{aligned}
& c_{1}^{*} 1^{2}+c_{2}^{*} 2^{2}+c_{3}^{*} 3^{2}=1 \\
& c_{1}^{*} 1^{3}+c_{2}^{*} 2^{3}+c_{3}^{*} 3^{3}=2 \\
& c_{1}^{*} 1^{4+k}+c_{2}^{*} 2^{4+k}+c_{3}^{*} 3^{4+k}=4+k-1
\end{aligned}
$$

Solving this system of equations for $C_{3}^{*}$ with Cramer's rule:

$$
C_{3}^{*}=\frac{\left|\begin{array}{ccc}
1^{2} & 2^{2} & 1 \\
1^{3} & 2^{3} & 2 \\
1^{4+k} & 2^{4+k} & 3
\end{array}\right|}{\left|\begin{array}{ccc}
1^{2} & 2^{2} & 3^{2} \\
1^{3} & 2^{3} & 3^{3} \\
1^{4+k} & 2^{4+k} & 3^{4+k}
\end{array}\right|}=\frac{1^{2} 2^{2}}{1^{2} 2^{2} 3^{2}} \frac{\left|\begin{array}{ccc}
1^{0} & 2^{0} & 1 \\
1^{1} & 2^{1} & 2 \\
1^{2+k} & 2^{2+k} & 3
\end{array}\right|}{\left|\begin{array}{ccc}
1^{0} & 2^{0} & 3^{0} \\
1^{1} & 2^{1} & 1 \\
1^{2+k} & 2^{2+k} & 3^{2+k}
\end{array}\right|}
$$

The question is, Can the following two expressions be equal to each other? 


$$
\frac{\left|\begin{array}{ccc}
1^{0} & 2^{0} & 1 \\
1^{1} & 2^{1} & 2 \\
1^{2} & 2^{2} & 3
\end{array}\right|}{\left|\begin{array}{lll}
1^{0} & 2^{0} & 3^{0} \\
1^{1} & 2^{1} & 3^{1} \\
1^{2} & 2^{2} & 3^{2}
\end{array}\right|} \leq \frac{\left|\begin{array}{ccc}
1^{0} & 2^{0} & 1 \\
1^{1} & 2^{1} & 2 \\
1^{2+k} & 2^{2+k} & 3
\end{array}\right|}{\left|\begin{array}{ccc}
1^{0} & 2^{0} & 3^{0} \\
1^{1} & 2^{1} & 1 \\
1^{2+k} & 2^{2+k} & 3^{2+k}
\end{array}\right|}
$$

Direct computation yields the following relation between $c_{3}$ and $c_{3}^{*}$ :

$$
2^{4+k} \leq 3^{2+k}+2 k+7
$$

Here the equality sign only stands for $k=0$. If $k>0$, the strict inequality holds:

$$
2^{4+k}<3^{2+k}+2 k+7
$$

This proves that $c_{3}$ differs from $c_{3}^{*}$ for all $k>0$.

The same proof applies for $c_{1}, c_{2}, c_{4}, c_{5}, \cdots, c_{n}$.

Consequently, the infinite system of equations is inconsistent. Q.E.D.

\section{Conclusions}

My main goal was to draw attention to the advantages of the factorial series and the factorial number system in the study of irrational and transcendental numbers.

The periodic representation makes it easier to inquire into these properties.

Proving the transcendence of e by mostly elementary methods used in this paper may help to prove the transcendence of other real numbers with relative ease.

Some problems in the Diophantine approximation of irrationals [10] can better be studied among periodic numbers.

Proposals to proceed:

I could not apply these methods to other important numbers like $\pi$, or Riemann numbers. Perhaps other techniques in calculus can provide the missing links.

I studied the digits of Egyptian fractions in the factorial number system with the help of Salát's software [11]. I had some interesting findings which can be worth developing.

I have not inquired into the properties of integers in this paper, but elementary calculations show that the periodic property can be extended to certain integers as well.

\section{Acknowledgments}

This work is dedicated to the memory of my brilliant student and good friend Máté Salát (1984-2009).

\section{Conflicts of Interest}

The author declares no conflicts of interest regarding the publication of this paper. 


\section{References}

[1] Agarwal, R. (2021) Pythagoreans Figurative Numbers: The Beginning of Number Theory and Summation of Series. Journal of Applied Mathematics and Physics, $\mathbf{9}$, 2038-2113. https://doi.org/10.4236/jamp.2021.98132

[2] Dickson, L.E. (1919) History of the Theory of Numbers. Carnegie Institution of Washington, Vol. 1, 263-278. https://doi.org/10.5962/t.174912

[3] Zaid, Y., Shiha, F. and El-Desouky, B. (2020) Generalized Central Factorial Numbers with Odd Arguments. Open Journal of Modelling and Simulation, 8, 61-72. https://doi.org/10.4236/ojmsi.2020.83005

[4] Sierpiński, W. (1988) Elementary Theory of Numbers. Schinzel. Ch. VII, A., Ed., 2nd Edition, North-Holland \& Polish Scientific Publishers, Amsterdam.

[5] Wikipedia: Factorial Number System. http://en.wikipedia.org/wiki/Factorial_number_system

[6] Jones, W.B. and Thorn, W.J. (1984) Continued Fractions (Analytic Theory and Application), Encyclopedia of Mathematics and Its Applications. Vol. 11. Cambridge University Press.

[7] Lambert, J.H. (2004) [1768] Mémoire sur quelques propriétés remarquables des quantités transcendantes circulaires et logarithmiques. In: Berggren, L., Borwein, J.M. and Borwein, P.B., Eds., Pi, A Source Book, 3rd Edition, Springer-Verlag, New York, 129-140. https://doi.org/10.1007/978-1-4757-4217-6_18

[8] Lénárt, I. (1993) Alternative Models on The Drawing Ball. Educational Studies in Mathematics, 24, 277-312. https://doi.org/10.1007/BF01275428

[9] Faber, G. (1905) Über die Anzahlbarkeit der Rationalen Zahlen. Mathematische Annalen, 60, 196-203. https://doi.org/10.1007/BF01677267

[10] Freud, R. and Gyarmati, E. (2000) Számelmélet (Theory of Numbers, in Hungarian). Nemzeti Tankönyvkiadó, 348-355.

[11] Salát, M. (2007) Software for Converting Numbers Into the Factorial Number System. 